by experiments and FEA. Most of the V-shape scratches developed into overlap defects after rolling. The scratch depth at a free surface, where the scratch does not come into contact with a roll, increases, whereas the scratch depth decreases when scratches come into contact with the roll and are subjected to compressive stress. In the case of wire drawing, when the scratch angle is small, the scratch side surfaces are pushed toward each other and the scratch becomes an overlap defect. In contrast, when the scratch angle is large, the bottom of the scratch rises, and the scratch is recovered satisfactorily. Furthermore, the scratch shape and the drawing conditions were varied, and the deformation state of a scratch was clarified.

\section{PLF-14: FE Simulation on Blank Holder Con- trol Using Friction Reducing Algorithm in Deep Drawing Process}

K. MANABE, Y. YAMAUCHI, T. YAGAMI, Tokyo Metropolitan University,

E-mail: manabeQcomp.metro-u.ac.jp

Recent global efforts for environmental issue are also required in sheet metal forming. For the reason, some research projects have been conducted on unlubricated process as well as on use of volatile lubricants. However, low formability due to worse lubrication conditions is still a matter. In this study, in order to improve the friction conditions, a new algorithm for controlling blank holding force (BHF) and punch speed (SPD) was proposed. The concept is to separate wrinkle eliminating process from drawing process. In other words, the process proceeds with an extremely low BHF until a wrinkle grows enough, and the wrinkle is eliminated by BHF loading without punch penetration. Its effectiveness was investigated by finite element (FE) simulation, in which two blank models $(0.5 \mathrm{~mm}$ and $1.0 \mathrm{~mm}$ thickness) were used. As a result, the maximum forming forces decreased by $5.33 \%(0.5 \mathrm{~mm})$ and $1.55 \%(1.0 \mathrm{~mm})$ compared with uncontrolled model at constant minimum BHF. In addition, thinner blank sheet is better for the algorithm on the basis of the result that a cup height lowers and a thickness distribution shifts to be thick in the case of $0.5 \mathrm{~mm}$ thickness.

\section{PLF-15: Development of Sheet Hydroforming Simulation Program by Static Finite Element Method}

T. HAMA, M. ASAKAWA, T. HATAKEYAMA, H. UTSUGI, Waseda University; A. MAKINOUCHI, The Institute of Physical and Chemical Research; H. AMINO, Y. LU, Amino Corporation,

E-mail: HIROOOOOONQSUOU.WASEDA.JP

This paper describes the development of a sheet hydroforming simulation program. Several new algorithms for the treatment of hydraulic pressure have been developed and implemented into the static-explicit finite-element method code for tube hydroforming simulation which has been developed by the authors. Simulations of an ellipse deep drawing have been performed and the simulated results were compared with those of experiment. In the experimental result, the local thinning was observed not only at the punch shoulder but also at the upper part of the side wall. In the simulated result, although the quantitative agreement at the upper part of the side wall with the experiment could not be obtained, this trend of the thickness distribution was well reproduced. Moreover, the region where the local thinning occurred in the simulated result well corresponded to that of the fracture site observed in the experiment, demonstrating the validity of the developed code.

\section{PLF-16: Development of Thread Rolled Anti- loosening Bolts Based on the Double Thread Mechanism and a Performance Evaluation}

T. TAKEMASU, H. MIYAHARA, Kyushu University,

E-mail: gmzkckk@mech.kyushu-u.ac.jp

It has already been proven that bolt fasteners based on the double thread mechanism have an excellent anti-loosening performance. The purpose of this study is to establish a mass production method for these double thread bolts (DTBs) by thread rolling. The pitch ratio of the coarse thread and the fine thread of the target DTB is set as 2 to 1 . A two-die roller with a plunge feed is employed as the rolling method due to its fine processing precision. The roller die used in the experiments has special grooves on the external surface which follow the same outline as the thread profiles of the DTB. Using these special dies, the DTB can be successfully formed in the same process as single thread bolts. In comparison with the cutting DTB, the surface finish of the rolled DTB was much smoother and the grooves of the fine screw thread of the rolled DTB were more clearly marked on the coarse screw thread. The deformation of a workpiece during rolling is examined, and the examination shows that the formed material smoothly fills the die grooves in each cross section. The rolled DTBs completely pass the vibration loosening test with an extremely severe impact, as specified in NAS3354. Neither a crack nor any damage was observed after the loosening test. The tensile fatigue strength of the rolled DTB increased to $100 \%$ greater than that of the cutting DTB.

\section{PLF-17: Elastic Deformation of the Deep Drawing Die Supported by Multi Point}

T. OHASHI, National Institute of Advanced Industrial Science and Technology (AIST),

E-mail: tohashi@ni.aist.go.jp

The author suggests a multi-point support system for drawing dies. It enables a user to adjust the deformation of the die during the deepdrawing trial process, instead of correcting the die surface after the trial. The die is supported at multiple points by elastic cylindrical cells with ball contacts. Each cell has strain gauges on its surface and works as both a structural part of the die support and as a load cell. Therefore, the user can inspect changes in load and displacement at each supported point as the cell undergoes elastic deformation. The user can change the distribution of these supporting points in response to the results of the trial process and to the measured load or displacement. For example, when the all cells indicate the same load, the difference in the displacement of the supported points is close to zero. Thus, the die back will remain flat. The author calls the suggested a smart die structure and suggests its usage in comparing 3D solid data for a die with the results of 3D shape measurement of work pieces, or with the results of simulated deformation of the work piece. The aim of this study is to shorten the lead time for press working by accelerating the trial loop and by replacing an expert's skillful "correct and try" procedure with a recordable "adjust and try" process. In this paper, the author reports on software applications for the relocation procedure, and on the experimental results of strain change along the relocation on the surface of the die.

\section{PLF-18: Springback Control of Sheet Metal Air Bending Process}

\author{
J. WANG, S. VERMA, R. ALEXANDER, Texas A\&M University, \\ E-mail: jwang@tamu.edu
}

In air bending, it has been observed that the final bend angle, which is the angle achieved upon removal of the punch, is smaller than the initial bend angle. This springback is due to the elastic recovery of the sheet metal. Various theoretical models have been proposed to predict the springback using the tooling geometry and the known properties of the sheet metal. However, in a production environment, the actual properties of any given workpiece may vary from the rated properties of the lot. This variation causes the actual springback, which is due to actual properties, to deviate from the theoretical predictions. This paper presents an approach to estimate the workpiece properties and control punch displacement to achieve more accurate final bend angles. The proposed methodology involves measuring loaded and unloaded angles in incremental bending operations, characterizing workpiece properties from measured angles, and determining required punch position to obtain the desired final angle after springback. It was found that the proposed method can better predict springback and effectively reduce the variation 